Classification

Physics Abstracts

$61.16 \mathrm{P}-68.20-73.40 \mathrm{~N}$

\title{
Characterization of the metal-semiconductor interface by ballistic electron emission microscopy
}

\author{
Roland Coratger, François Ajustron and Jacques Beauvillain \\ CEMES-LOE / CNRS, 29 rue J. Marvig, BP. 4347, 31055 Toulouse, France
}

(Received 20 August, 1993, accepted 22 October, 1993)

\begin{abstract}
Résumé . - A travers les résultats obtenus sur des jonctions $\mathrm{Au}-\mathrm{Si}(100)$, nous présentons les principales caractéristiques de la Microscopie par Emission d'Electrons Balistiques (BEEM). La spectroscopie permet d'obtenir la hauteur de barrière Schottky locale avec une résolution de l'ordre du nanomètre à l'interface. La spectroscopie en mode "polarisation inverse" met en évidence un courant de même sens qu'en polarisation directe. Ce courant peut être interprété en considérant que la diffusion par interaction entre porteurs est le phénomène dominant dans le transport d'électrons de faibles énergies. De plus, les variations du courant collecteur permettent l'imagerie BEEM. La hauteur de barrière Schottky étant constante sur ces échantillons, le contraste des images BEEM est principalement attribué à la diffusion dans le film métallique et à la qualité de l'interface.
\end{abstract}

\begin{abstract}
Through the results obtained on Au-Si(100) junctions, we show the main aspects of Ballistic Electron Emission Microscopy (BEEM). Spectroscopy allows the local Schottky barrier height to be yielded with a lateral resolution at the interface in the nanometer range. A collector current of electrons is evidenced in the reverse voltage condition. Carrier-carrier scattering is assumed to account for this phenomenon and appears as the dominant process in low energy electron transport. In addition, collector current variations allow BEEM imaging. As the Schottky barrier height is found to be constant in these Au-Si(100) junctions, BEEM image contrast is mainly attributed to scattering in the gold film and interface quality.
\end{abstract}

\section{Introduction.}

Since its invention in 1981, the Scanning Tunneling Microscopy has proven to be a suitable tool for surface investigation [1]. Indeed, the exponential dependence of tunneling current in terms of tip-sample distance allows determination of the surface atomic structure with a resolution better than $0.1 \mathrm{~nm}$. However, the informations only concern the first surface layers or, at the most, the layer just below the surface for certain reconstructions [2].

This lack has been partially filled after the pioneering work of Kaiser and Bell in 1988 [3, 4]. These authors have used a new technique, Ballistic Electron Emission Microscopy (BEEM), to study the buried interface between a metal and a semiconductor, i.e. a Schottky junction. This 
Metal-Semiconductor (MS) contact leads to the well-know Schottky barrier which height $\mathrm{V}_{\mathrm{B}}$ may be estimated in a rough approximation as the metal work function $\Phi_{M}$ minus the electron affinity of the semiconductor $X_{\mathrm{S}}$. The tip of the STM is then used as a very localized electron or hole source according to the voltage polarity. The carriers ballistically injected in a thin metal layer may overcome the potential barrier at the interface, provided their incident energy is sufficient and the metal thickness is comparable to the electron mean free path in the bulk material (typically a few tens of nanometers for a few eV).

BEEM spectroscopy therefore consists of the current detection in the semiconductor $\left(I_{\mathrm{c}}\right)$ in terms of bias voltage $V$ at a constant position $(x, y)$ on the metal surface. The collector current is different of zero when $V$ is larger than $V_{\mathrm{B}}$ and the threshold in the $I_{\mathrm{c}}(V)$ spectrum gives the local value of $V_{\mathrm{B}}$. An other possible mode of working is BEEM imaging. In this case, the tip-sample voltage is kept constant at a value above the threshold. While scanning the metal surface with the tip, the vertical height of the tip and the local variations of the collector current in the semiconductor are simultaneously recorded. A BEEM image consists of a grey scale representation of the current local variations.

An other interesting aspect is the possible study of transport mechanism in thin metal layers. Indeed, the local variations of $I_{\mathrm{c}}$ may originate from several mechanisms: scattering in the metal layer, interface defects, variations of the mean free path in terms of electron energy, injection into the different minima of the semiconductor conduction band, ... that may be also studied with a great accuracy by this method.

In this paper, we illustrate the possibilities of BEEM through the results obtained on thin gold films evaporated on $\mathrm{Si}(100)$. We discuss the effects of the electron scattering in the gold film and at the interface. Spectroscopy with reverse bias voltages also allows direct characterization of carrier-carrier scattering. BEEM images illustrate the non-homogeneity of these peculiar heterojunctions.

\section{Experimental.}

The experiments have been carried out on a conventional home-made pocket size STM described elsewhere [5]. The experimental setup is described in figure 1. The bias voltage is established between the tip and the metal surface. This voltage may be kept constant (positive or negative) or may be variable with range, frequency and sign fixed by the user. A current-voltage converter allows the measurements of the collector current with an accuracy in the picoampere range. The total electrical noise of this setup is usually less than $1 \mathrm{pA}$.

The $\mathrm{Si}(100)$ substrate used in the experiments is of the $n$ type with a doping of $2 \times 10^{15} \mathrm{~cm}^{-3}$. After HF cleaning and desionized water washing of the silicon, the wafer is introduced in the preparation chamber where the vacuum is kept in the $10^{-8}-10^{-9}$ torr range. An AuSb ohmic contact is achieved on the back of the substrate to allow collector current detection. The Au films are then deposited with a thickness ranging from 5 to $12.5 \mathrm{~nm}$. An electrical contact is made before each BEEM observation by bonding a small $50 \mu \mathrm{m}$ diameter copper wire on a corner of the gold film. 

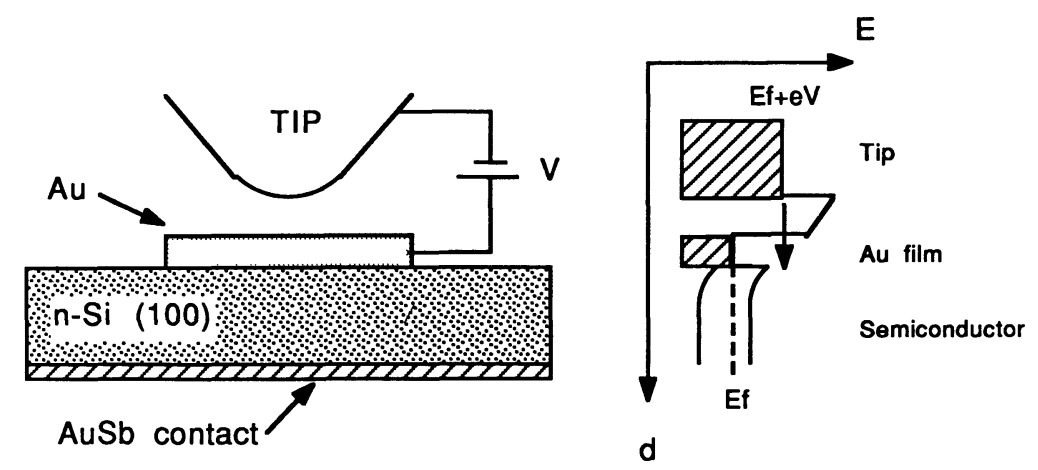

Fig. 1. - Schema of the experimental setup with the STM tip and the Metal-Semiconductor junction. The drawing in the right-hand gives the energy diagram when the sample voltage is positive and larger than the Schottky barrier height at the interface.

\section{Results.}

\subsection{SPECTROSCOPY.}

3.1.1 Positive sample voltage. - The doping of the semiconductor gives the sign of the potential step at the interface and then, the carrier type: electrons or holes. For $n$ type silicon, only electrons may overcome the Schottky barrier. When the sample bias voltage is positive, electrons from the tip in the $E_{\mathrm{F}}$ to $E_{\mathrm{F}}+\mathrm{eV}$ energy window are injected in the metal as represented in the right of figure 1. Current in the semiconductor $I_{\mathrm{c}}$ is therefore zero when $V$ is less than $V_{\mathrm{B}}$ and increases when the voltage exceeds $V_{\mathrm{B}}$.

Figure 2a shows a spectrum achieved at a constant lateral position on the Au surface with a tunneling current of $3 \mathrm{nA}$. As expected, the curve shows a characteristic threshold around $0.8 \mathrm{eV}$. Below this value, the collector current is nearly zero $\left(I_{c}\right.$ is equal to the noise of all the setup, i.e. around $1 \mathrm{pA}$ ). Beyond $0.8 \mathrm{eV}, I_{\mathrm{c}}$ increases and reaches $100 \mathrm{pA}$ for $V=1.6$ volts. Figure $2 \mathrm{~b}$ is the derivative of figure $2 \mathrm{a}$. These two curves illustrate the behavior of injected electrons when their energy exceeds the threshold. On the one hand, the derivative is quasi linear between 0.8 and $1.1 \mathrm{~V}$ which suggests a square law behavior of the collector current as a function of voltage above threshold. On the other hand, the spectrum slope tends to decrease above $1.5 \mathrm{~V}$ and the original data reach a saturation at $2.4 \mathrm{~V}$. This is clearly evidenced in the derivative curve. In some case, negative slopes are evidenced for voltages up to $2.2 \mathrm{~V}$.

Other experiments demonstrate the linear dependance of the collector current $I_{\mathrm{c}}$ in terms of tunneling current $I_{\mathrm{t}}$. Four spectra have been taken in the same place of the sample but with four different tunneling currents: $1,2,3$ and $4 \mathrm{nA}$. For three bias voltages $1,1.2$ and $1.4 \mathrm{~V}$, we have represented $I_{\mathrm{c}}$ in terms of $I_{\mathrm{t}}$ (Fig. 3). The three curves unambiguously show the expected linear dependence: the number of collected electrons is therefore proportional to the total number of injected carriers.

3.1.2 Negative sample voltage. - In this case, the electron transfer takes place from the Au surface to the STM tip. Holes are therefore injected in the metal layer. Figure 4 is a spectrum taken with a tunneling current of $20 \mathrm{nA}$ in order to increase the signal to noise ratio. It is important to observe that the collector current $I_{\mathrm{c}}$ has the same sign: we do detect a current of electrons in the semiconductor. Secondary electrons are therefore created and collected in the semiconductor. The spectrum always presents a threshold around $0.8 \mathrm{eV}$ but the current increase is weaker beyond 


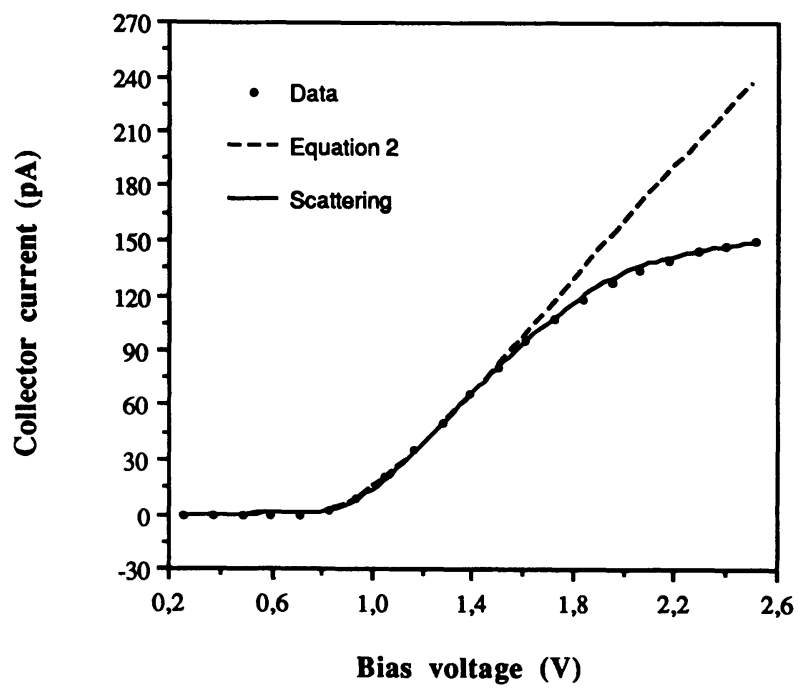

a)

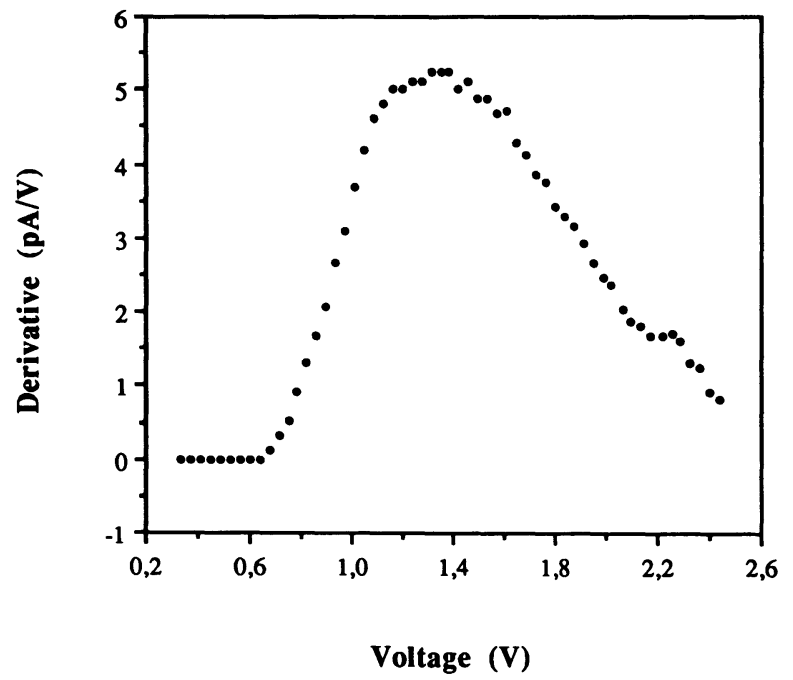

b)

Fig. 2. - a) Ballistic current in terms of sample bias voltage for a gold thickness of $7.5 \mathrm{~nm}$ and a tunneling current of $3 \mathrm{nA}$ (black marks). Only every third point from the original data set is shown. The dotted line results from a calculation using equation (2) with $R=0.162 \mathrm{eV}^{-1}$ and $V_{\mathrm{B}}=0.74 \mathrm{~V}$. The introduction of scattering in equation (2) accounts for the saturation of the collector current observed above $1.5 \mathrm{~V}$ (solid line with $V_{\mathrm{B}}=0.73 \mathrm{~V}$ ). b) Derivative of the experimental data of figure 2a. A quasi-linear variation of this derivative may be evidenced above the threshold voltage $(\approx 0.8 \mathrm{~V})$. The decrease of the curve above $1.5 \mathrm{~V}$ illustrates the tendency towards saturation of the experimental spectrum.

this value: $I_{\mathrm{c}}$ is equal to $70 \mathrm{pA}$ at $-1.7 \mathrm{~V}$. In the same conditions (i.e. $I_{\mathrm{t}}=20 \mathrm{nA}$ ), $I_{\mathrm{c}}$ is ten times larger when the sample voltage is positive $(V=+1.7 \mathrm{~V})$. 


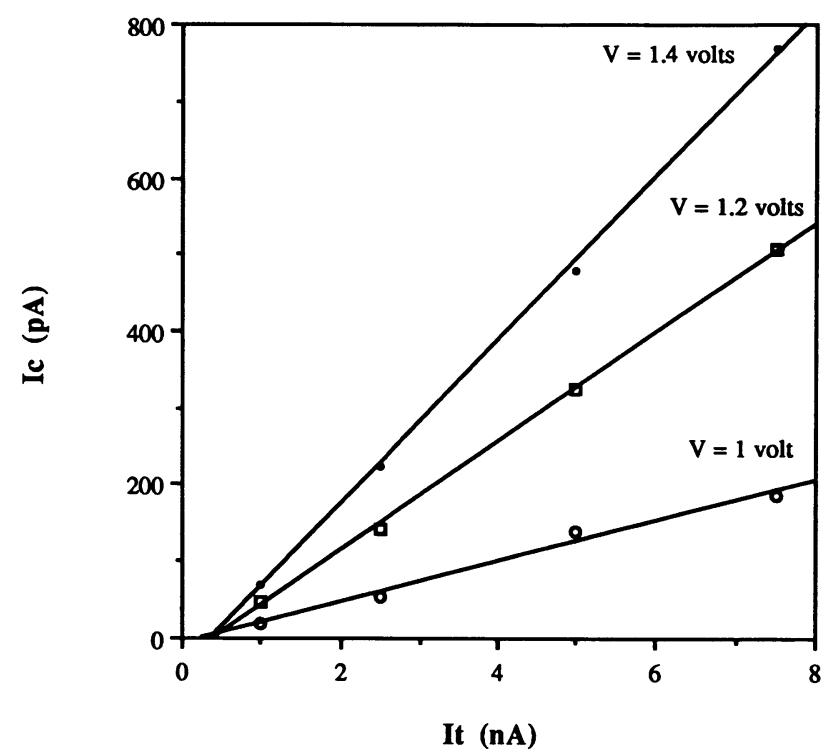

Fig. 3. - Variations of $I_{\mathrm{c}}$ in terms of $I_{\mathrm{t}}$ for three bias voltages: $1,1.2$ and $1.4 \mathrm{~V}$ deduced from four spectra taken at the same place on the Au surface.

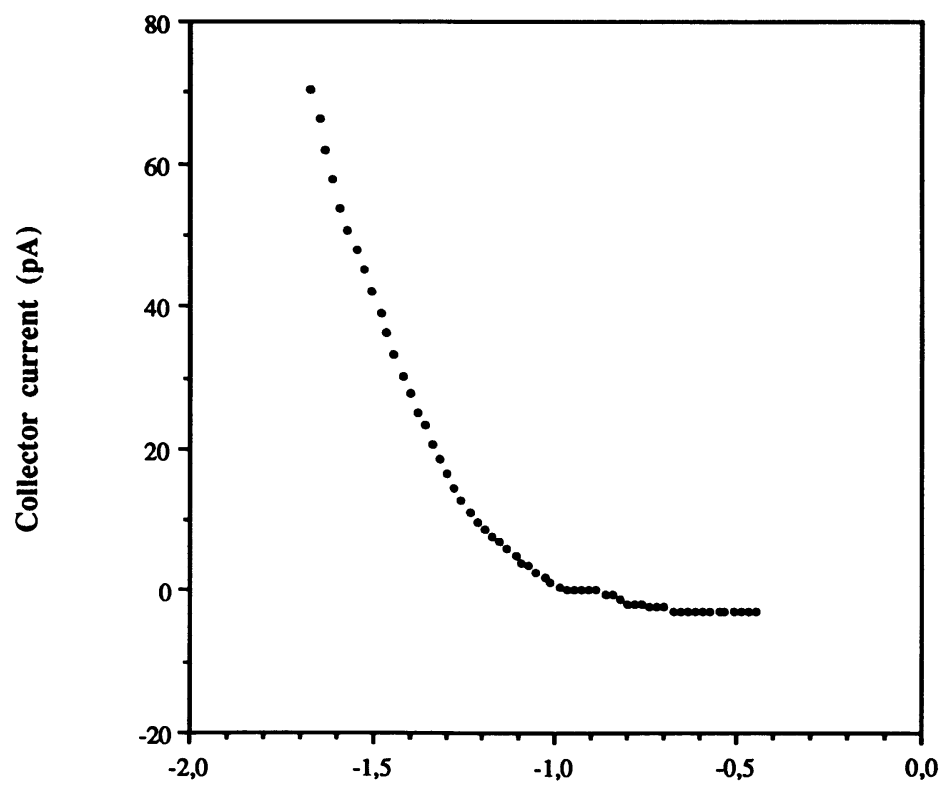

Sample bias voltage (V)

Fig. 4. - Experimental collector current in terms of negative sample tunnel voltage. The tunneling current is equal to $20 \mathrm{nA}$ and the gold thickness is $12.5 \mathrm{~nm}$. The sign of the current indicates that "secondary" electrons are collected in the semiconductor. 
3.2 BEEM IMAGING. - The BEEM images give the local variations of the collector current while scanning the metal surface with the STM tip at a constant tunneling current and by applying a constant bias voltage between tip and sample. This voltage is above the threshold value $V_{\mathrm{B}}$. Two images are simultaneously stored: the topography of the Au surface $z(x, y)$ and the collector current variations $I_{\mathrm{c}}(x, y)$.

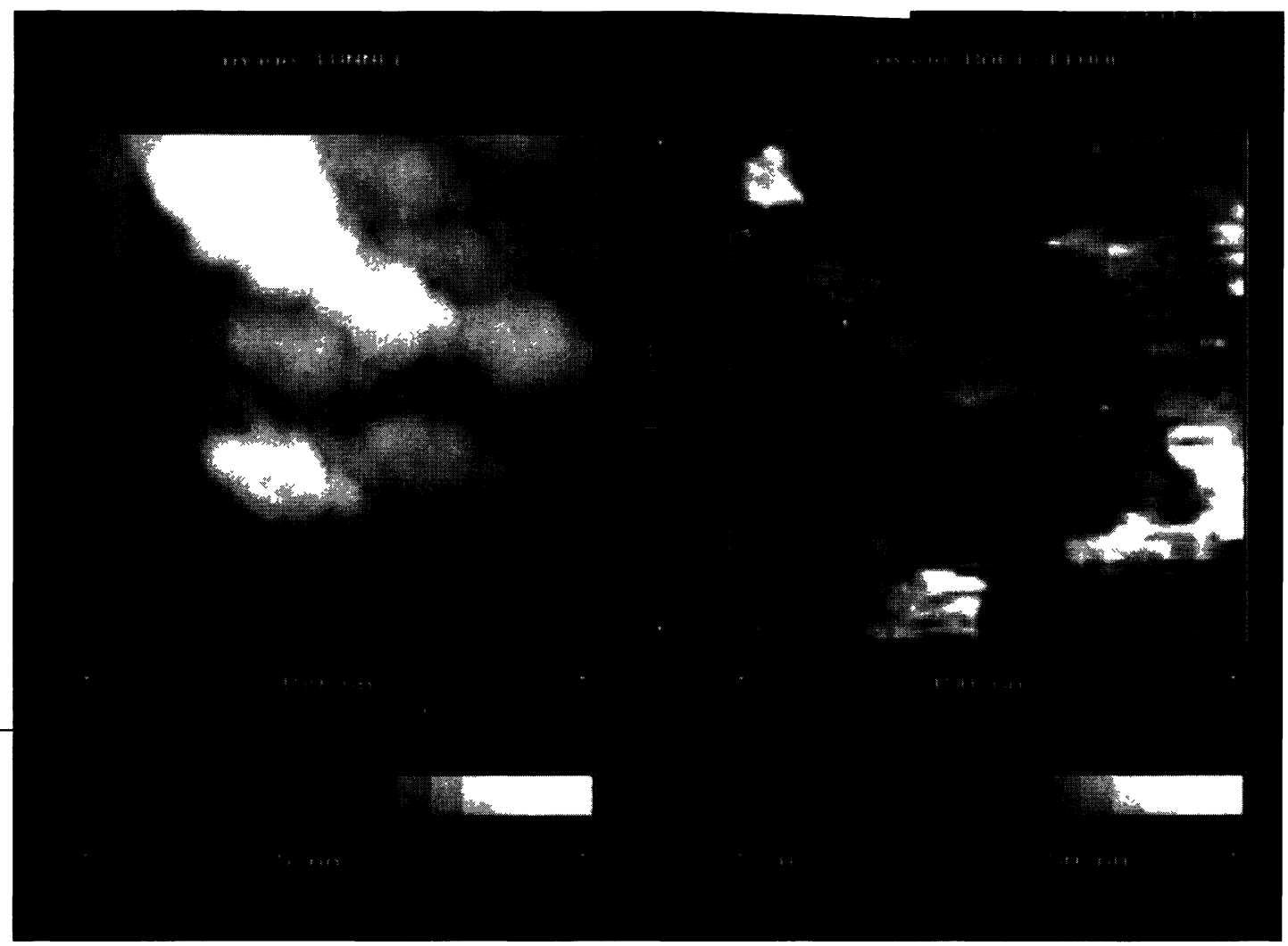

Fig. 5. - STM topography (left) and BEEM image (right) of the Schottky junction with a $7.5 \mathrm{~nm}$ gold thickness. Maximum contrast of the BEEM image corresponds to collector current variations of $50 \mathrm{pA}$.

Figure 5 is a BEEM image $\left(150 \times 150 \mathrm{~nm}^{2}\right)$ of a Schottky junction with a gold thickness of 7.5 $\mathrm{nm}$, a tunneling current of $5 \mathrm{nA}$ and a bias voltage of $1.4 \mathrm{~V}$. The left image is the STM image. The STM topography shows that the gold surface is made of hillocks and valleys with a maximum corrugation of $5 \mathrm{~nm}$. The BEEM image looks like the topography because the large domains of low or high injection yield follow the grain boundaries. However, several adjacent grains may give the same contrast in BEEM experiments. This first result is already surprising: this MS junction gives some differences between topography and ballistic results. BEEM contrast is not simply related to surface topography as suggested in previous papers [6].

The Au thickness also plays a low role in BEEM contrast. Indeed, grains with high corrugations may give the higher injected currents. This is probably due to the small thickness of gold as 
compared to the mean free path of electrons. It first means that the yield of injection cannot be simply explained by film thickness variations.

\section{Discussion.}

Ballistic Emission Electron Microscopy allows determination of buried MS interfaces with a high resolution. Between the STM tip and the gold surface, we know that electrons are injected with a small angle relative to the surface normal. However, all these electrons contribute to the tunneling current $I_{\mathrm{t}}$. This is completely different for the collector current $I_{\mathrm{c}}$. On the one hand, these electrons should have a sufficient kinetic energy to overcome the potential step and to be collected in the semiconductor $\left(\mathrm{eV}>\mathrm{eV}_{\mathrm{B}}\right)$. On the other hand, limitations on transverse momentum conservation lead to reflection of electrons whose transverse momentum component $\mathbf{k}_{/ /}$(i.e. parallel to the interface) is too large (with $\mathbf{k}_{\text {electron }}=\mathbf{k}_{\perp}+\mathbf{k}_{/ /}$). This effect is illustrated in figure 6. Path " 1 " corresponds to an electron which $\mathbf{k}_{/ /}$component is relatively small. This electron is refracted in the semiconductor and participates to $I_{\mathrm{c}}$. The trajectory " 2 " is associated to an electron with large $\mathbf{k}_{/ /}$. This electron is reflected in the metal layer. The case " 3 " is also impossible: either the multiple inelastic interactions have considerably reduced the incident energy of this carrier or the covered distance with only elastic scattering is larger than its mean free path in the bulk.

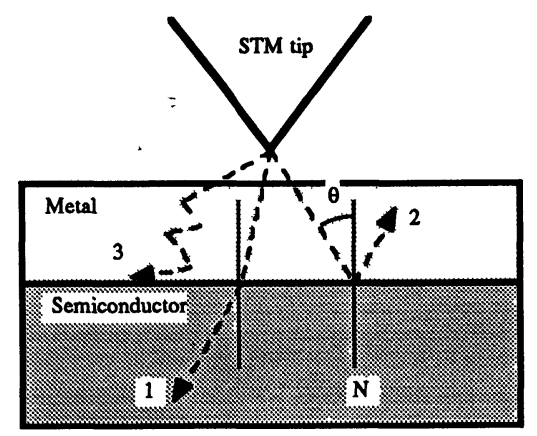

Fig. 6. - Different possibilities for electron injection at the Metal-Semiconductor interface. Path 1 corresponds to an electron refracted in the semiconductor because its $k_{/ /}$is relatively small. Electrons 2 (k// is too large and $\theta>\theta_{c}$ ) and 3 (multiple interactions) remain in the semiconductor.

This effect could be taken into account by calculating the critical angle $\theta_{\mathrm{c}}$ between the incident electron path and the normal to the interface $N$ (see Fig. 6). This angle is given by the following relation [4]:

$$
\frac{\sin ^{2} \theta_{\mathrm{c}}=\frac{m_{\mathrm{t}}}{m} e\left(V-V_{\mathrm{B}}\right)}{E_{\mathrm{F}}+e V}
$$

where $m_{\mathrm{t}}$ is the electron effective mass parallel to the interface within the semiconductor, $m$ is the free electron mass, $E_{\mathrm{F}}$ is the Fermi energy and $V$ the tip-sample voltage.

For silicon in which $m_{\mathrm{t}}$ is equal to $0.1 \mathrm{~m}$, we obtain a critical angle of $5^{\circ} 50$ for $V=1.5$ volts. This angle is therefore relatively small and explains the resolution of this new microscopy: for an Au thickness of $10 \mathrm{~nm}$, the resolution at the interface is $1.7 \mathrm{~nm}$. Each spectrum gives the local 
electronic characteristic of the MS interface with this high resolution. The same properties apply to the BEEM images.

Interpretation of the experimental spectra has been furnished by Kaiser and Bell in their first paper on BEEM [3]. This one dimensional theoretical model has been later improved by two dimensional calculations [4]. The authors have used the formalism, introduced by Simmons in 1963 for tunneling between planar electrodes [7], to describe both tunneling and collector current. Hence, the collector current may be written as:

$$
I_{\mathrm{c}}=R C \int_{E_{\min }}^{\infty} D\left(E_{\perp}\right) \int_{0}^{E_{\max }} f(E) \mathrm{d} E_{/ /} \mathrm{d} E_{\perp}
$$

where $C$ is a constant, $E_{\perp}$ and $E_{/ /}$are the energy perpendicular and parallel to the interface respectively, $f(E)$ is the Fermi-Dirac function and $D\left(E_{\perp}\right)$ is the well-known expression for the transmission probability of a square barrier. $E_{\min }$ and $E_{\max }$ are given by the energy and momentum matching conditions at the interface. $R$ is a measure of attenuation due to scattering in the metal layer. In the calculations, the collector current only depends on two parameters: the Schottky barrier height $V_{\mathrm{B}}$ and $R$.

We have used this formalism to fit the experimental data in the case of low bias voltages, i.e. at the most $0.5 \mathrm{~V}$ above the threshold. The dotted line in figure $2 \mathrm{a}$ is a curve calculated by this formalism with $R=0.162 \mathrm{eV}^{-1}$ and $V_{\mathrm{B}}=0.74 \mathrm{~V}$. As expected, the model accounts for the spectroscopic results in the low voltage case. Kaiser et al. [4] have also demonstrated the calculated spectra have a square law behavior just above threshold as observed in the derivative of figure $2 \mathrm{~b}$.

Beyond these voltage values, equation (2) is less accurate: the calculated values are greater than the experimental data (see Fig. 2a above $1.5 \mathrm{~V}$ ). The tendency to reach saturation in the experimental spectra has been theoretically studied by Prietsch and Ludeke $[8,9]$. These authors add in equation (2) a function that accounts for the variations with energy of the electron mean free path (we have to kept in mind that $R$ is an energy independent constant in (2)). Indeed, it has been shown in previous works that, in the $\mathrm{eV}$ range, the mean free path $\lambda \mathrm{e}$-e due to electronelectron scattering decreases when the energy increases [10]. In figure 2a, the solid line has been calculated after introduction of inelastic scattering in the gold film in the form of an exponential function. This example underlines the abilities of BEEM to study the transport properties of low energy electrons through thin metal layers.

More generally, we have used the simplest formalism (2) to fit all the spectra yielded during the experiments in the low energy range. Taken the accuracy of the method into account, i.e. around $50 \mathrm{meV}$, we find the Schottky barrier height is constant for this MS interface $(\approx 0.77 \mathrm{eV})$. The variations of $I_{\mathrm{c}}$ rather arise from the fluctuations of the $R$ parameter. This value is smaller than those usually obtained by BEEM on Si substrates [4] or by other techniques [11] (generally above $0.8 \mathrm{eV}$ ).

The same Schottky barrier height at the MS interface is still evidenced in the spectroscopy with negative sample voltages because there is no current below a certain voltage which is also equal to $e V_{\mathrm{B}}$. The mechanisms at the origin of these observations include carrier-carrier scattering in the $\mathrm{Au}$ layer. At negative sample voltage, a ballistic hole distribution is created in the gold film. Hot electrons may be produced by scattering of some holes with equilibrium electrons and collected in the semiconductor [12] (see Fig. 7). The presence of a collector current is only due to carriers which undergo scattering events. As this current may be relatively important ( $>50 \mathrm{pA})$, we can conclude that the carrier-carrier scattering appears to be the dominant interaction in transport throughout the thin metallic layer.

Previous works on Au-Si junctions present similar STM topographies but very uniform BEEM images $[4,6]$. Our observations show that the voltage threshold $V_{\mathrm{B}}$ is the same in all the images 


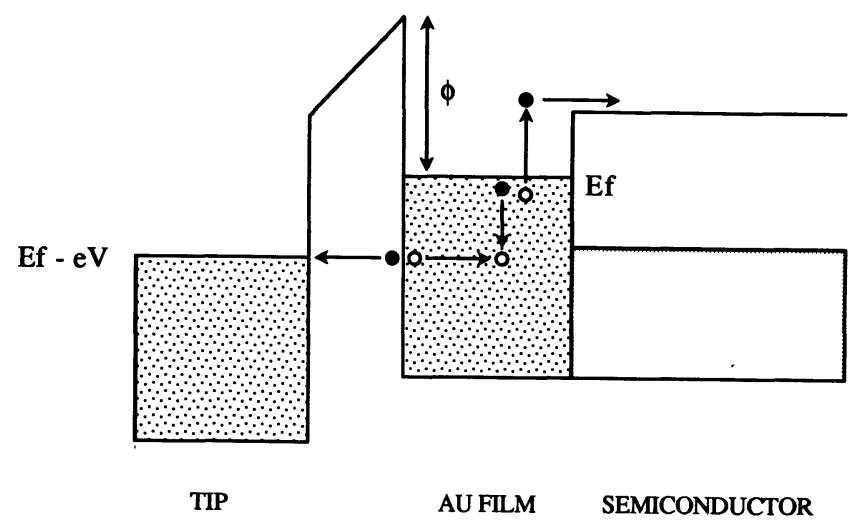

Fig. 7. - Energy diagram for the scattering process (sample voltage negative and $n$-type semiconductor). For simplicity, the semiconductor band bending at the interface has been neglected. A ballistic hole distribution is then created in the gold film. Hot electrons may be produced by scattering of some holes with equilibrium electrons and collected in the semiconductor.

and the injection yield locally varies which leads to the observed collector current variations. Some authors have suggested that this could be due to variations of the injection angle on the grain edges [9]. In our case, this interpretation is not very convincing because the domains have an uniform contrast even at the grain edges. Interface roughness may be invoked if one admits that adjacent grains in a BEEM domain are located in areas where the interface normal has the same direction (because the BEEM domains follow the grain boundaries).

We assume that the unusual large domains where low or high ballistic currents are observed, may be attributed to interface domains rather than to different surface properties. Interface defects and intermigration are assumed to play an important role in the variations of injection yield [13]. Oxides on the $\mathrm{Si}(100)$ substrate are undoubtedly present before gold deposition. This could account for the relative low barrier height observed during the spectroscopic measurements as reported in previous papers where techniques such as $I(V)$ or $C(V)$ measurements have been used [14]. As suggested in [15], no BEEM current is observed when the interface oxide layer is too thick. In this case, darker areas in the BEEM image could correspond to interface domains with thicker oxide layers. This oxide layer, that considerably decreases the electron mean free path at high kinetic energy, could also account for the saturation of the spectra at high voltage.

Interpretation of these BEEM images is therefore assumed to be tighly dependent of the interface quality, i.e. substrate cleanness before metal deposition, quality of the first metal layers,... Further experiments are necessary to resolve this problem. However, BEEM reveals the heterogeneity of the MS junction and then appears to be of fundamental importance for Schottky junction optimization.

\section{Summary.}

In this paper, we show the possibilities of Ballistic Electron Emission Microscopy for MetalSemiconductor interface characterization. Spectroscopy allows the Schottky barrier height to be determined with a lateral resolution at the interface in the nanometer range. For $\mathrm{Au}$ on $\mathrm{Si}(100)$, this barrier height is found to be constant with a value lower than usually obtained on similar junctions. Spectra realized in the reverse tip bias condition demonstrate that carrier-carrier scattering 
is the dominant energy loss mechanism in transport through thin metallic layers. As the barrier height is constant, BEEM images give the variations of the injection yield in the semiconductor. Large domains are mainly attributed to defects at the interface. All these results demonstrate that BEEM appears as one of the most suitable tool to study buried interfaces.

\section{Acknowledgements.}

We would like to thank G. Peuch for technical assistance and F. Rossel for sample preparation.

\section{References}

[1] Binnig G., Rohrer H., Gerber Ch., Weibel E., Appl. Phys. Lett. 40 (1982) 178.

[2] Binnig G., Rohrer H., Gerber Ch., Weibel E., Surf. Sci. 131 (1983) L379.

[3] Bell L.D., Kaiser W.J., Scanning Microsc. 2 (1988) 1231.

[4] Bell L.D., Kaiser W.J., Phys. Rev. Lett. 61 (1988) 2368.

[5] Coratger R., Beauvillain J., Ajustron F., Lacaze J.C., Tremollieres C., Rev. Sci. Instrum. 62 (1991) 830.

[6] Palm H., Arbes M., Schulz M., Appl. Phys. A56 (1993) 1.

[7] Simmons J.G., 34 (1963) 1793.

[8] Ludeke R., Prietsch M., Samsavar A., J. Vac. Sci. Technol. B9 (1991) 2342.

[9] Ludeke R., Prietsch M., J. Vac. Sci. Technol. A9 (1991) 885.

[10] Quinn J.J., Phys. Rev. 126 (1962) 1453.

[11] Brillson L.J., Surf. Sci. Rep. 2 (1982) 123.

[12] Bell L.D., Hecht M.H., Kaiser W.J., Davis L.C., Phys. Rev. Lett. 64 (1990) 2679.

[13] Cros A., Derrien J., Salvan F., Surf. Sci. 110 (1981) 471.

[14] Rhoderick E.H., Williams R.H. (Clarendon, Oxford Press, 1988).

[15] Hallen H.D., Fernandez A., Huang T., Buhrman R.A., Silcox J., J. Vac. Sci. Technol. B9 (1991) 585. 